PROCEEDINGS OF THE

AMERICAN MATHEMATICAL SOCIETY

Volume 126, Number 8, August 1998, Pages 2389-2397

S 0002-9939(98)04709-1

\title{
AN APPLICATION OF SCHAUDER'S FIXED POINT THEOREM WITH RESPECT TO HIGHER ORDER BVPS
}

\author{
FU-HSIANG WONG
}

(Communicated by Hal L. Smith)

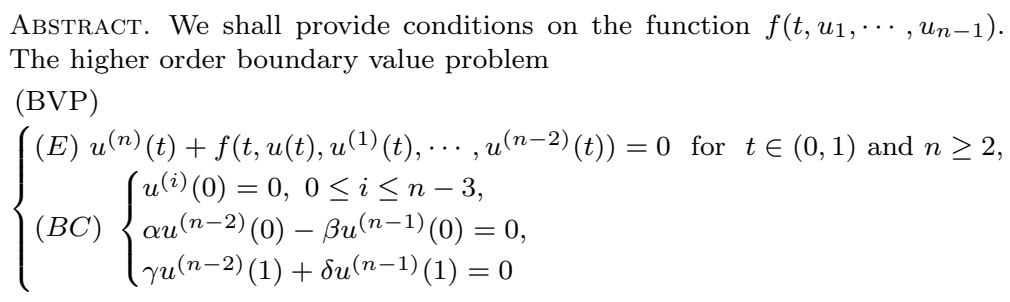

has at least one solution.

\section{INTRODUCTION}

In this article, we shall attempt to construct some existence criteria for the following $n$-th order boundary value problem:

(BVP)

$$
\left\{\begin{array}{l}
(E) u^{(n)}(t)+f\left(t, u(t), u^{(1)}(t), \cdots, u^{(n-2)}(t)\right)=0 \text { for } t \in(0,1) \text { and } n \geq 2, \\
(B C)\left\{\begin{array}{l}
u^{(i)}(0)=0,0 \leq i \leq n-3, \\
\alpha u^{(n-2)}(0)-\beta u^{(n-1)}(0)=0, \\
\gamma u^{(n-2)}(1)+\delta u^{(n-1)}(1)=0 .
\end{array}\right.
\end{array}\right.
$$

The motivation for the present work stems from many recent investigations in [1]-[3], [8], [13], [23]-[24]. In fact, particular cases of the boundary value problem (BVP) occur in various physical phenomena [4]-[7], [9]-[10], [13], especially such as gas diffusion through porous media, thermal self-ignition of a chemically active mixture of gases in a vessel [7], catalysis theory [9], chemically reacting systems, as well as adiabatic tubular reactor processes. For other related works, we refer to recent contributions of Agarwal and Wong [1]-[3], Anuradaha, Hai and Shivaji [4], Bailey, Shampine and Waltman [5], Erbe and Wang [13], Granas, Guenther and Lee [16], Lee and O'Regan [21], Chyan and Henderson [8], Henderson [17], Vasilev and Klokov [23] and Kelevedjiev [18]-[19] and the references therein.

Here, we shall remark that there are four main techniques to treat the existence of (BVP) as follows:

Received by the editors January 22, 1997.

1991 Mathematics Subject Classification. Primary 34B15.

Key words and phrases. Higher order boundary value problems, solution, operator equation, Green's function, Schauder's fixed point theorem, upper-lower-solutions.

(C)1998 American Mathematical Society 
Method 01. Shooting method ([25]). This method has been used successfully in the study of some special boundary value problems, if one can guarantee the uniqueness of related initial value problems.

Method 02. Fixed point index method ([1]-[2], [11], [20]). This method has many advantages in treating non-singular boundary value problems and relies on the following lemma:

Let $E$ be a Banach space, and let $C \subseteq E$ be a cone in $E$. Assume that $\Omega_{1}, \Omega_{2}$ are open subsets of $E$ with $0 \in \Omega_{1}, \overline{\Omega_{1}} \subset \Omega_{2}$, and let

$$
\left.T: C \cap \overline{\Omega_{2}} \backslash \Omega_{1}\right) \longrightarrow C
$$

be a completely continuous operator such that either

(i) $\|T u\| \leq\|u\|, u \in C \cap \partial \Omega_{1}$, and $\|T u\| \geq\|u\|, u \in C \cap \partial \Omega_{2}$; or

(ii) $\|T u\| \geq\|u\|, u \in C \cap \partial \Omega_{1}$, and $\|T u\| \leq\|u\|, u \in C \cap \partial \Omega_{2}$.

Then $T$ has a fixed point in $C \cap\left(\overline{\Omega_{2}} \backslash \Omega_{1}\right)$.

Method 03. Nonlinear alternative or topological method ([6], [15]-[16], [22]). This method was initiated in Granas, Guenther and Lee [15]-[16]:

Let $X, Z$ be real vector normed spaces, $L: \operatorname{dom} L \subset X \rightarrow Z$ a linear Fredholm mapping of index zero, $\Omega \subset X$ an open bounded subset, and $N: \bar{\Omega} \rightarrow Z$ an $L$ compact mapping. If $\operatorname{ker} L=\{0\}, 0 \in \Omega$ and

$$
L x-\mu N x \neq 0
$$

for every $(x, \mu) \in(\operatorname{dom} L \cap \partial \Omega) \times(0,1)$, then the equation

$$
L x=N x
$$

has at least one solution in $\operatorname{dom} L \cap \bar{\Omega}$.

Method 04. Schauder's or Barrier's method ([12]). In the next section, we attempt to establish a general existence principle for $(B V P)$, which relies on Schauder's fixed point theorem:

Let $C$ be a convex subset of a normed linear space $\mathbb{E}$. Then every compact continuous function $T: C \longrightarrow C$ has at least one fixed point.

\section{MAin ReSUlts}

Let $\alpha, \gamma, \beta, \delta \geq 0, \rho:=\gamma \beta+\alpha \gamma+\alpha \delta>0$ and $\mathbb{B}$ be the Banach space

$$
\left\{u \in C^{(n)}(0,1) \cap C^{(n-1)}[0,1] \mid u^{(i)}(0)=0,0 \leq i \leq n-3\right\}
$$

with norm $\|u\| \equiv \sup _{t \in[0,1]}\left|u^{(n-2)}(t)\right|$.

In order to abbreviate our discussion, we suppose throughout this paper that the following assumptions hold:

$\left(C_{1}\right): K(t, s)$ is the Green's function of the differential equation

$$
-u^{(n)}(t)=0 \text { in }(0,1)
$$

subject to the boundary conditions $(B C)$.

$\left(C_{2}\right): k(t, s)$ is the Green's function of the differential equation

$$
-u^{\prime \prime}(t)=0 \text { in }(0,1)
$$


subject to the boundary conditions

$\left(\mathrm{BC}^{*}\right)$

$$
\left\{\begin{array}{l}
\alpha u(0)-\beta u^{\prime}(0)=0 \\
\gamma u(1)+\delta u^{\prime}(1)=0
\end{array}\right.
$$

$\left(C_{3}\right): f \in C\left([0,1] \times \mathbb{R}^{n-1} ; \mathbb{R}\right)$.

$\left(C_{4}\right): v, w \in \mathbb{B}$ are lower solutions and upper solutions of (BVP) in the sense:

$$
\left\{\begin{aligned}
&\left(1^{0}\right) \quad v^{(n)}(t)+f\left(t, v(t), v^{(1)}(t), \cdots, v^{(n-2)}(t)\right) \geq 0 \text { for } t \in(0,1) \\
&\left(2^{0}\right) \quad w^{(n)}(t)+f\left(t, w(t), w^{(1)}(t), \cdots, w^{(n-2)}(t)\right) \leq 0 \text { for } t \in(0,1), \\
&\left(3^{0}\right) \quad\left\{\begin{array}{l}
v^{(i)}(0)=0,0 \leq i \leq n-3 \\
\alpha v^{(n-2)}(0)-\beta v^{(n-1)}(0) \leq 0 \\
\gamma v^{(n-2)}(1)+\delta v^{(n-1)}(1) \leq 0
\end{array}\right. \\
&\left(4^{0}\right) \quad\left\{\begin{array}{l}
w^{(i)}(0)=0,0 \leq i \leq n-3 \\
\alpha w^{(n-2)}(0)-\beta w^{(n-1)}(0) \geq 0 \\
\gamma w^{(n-2)}(1)+\delta w^{(n-1)}(1) \geq 0
\end{array}\right.
\end{aligned}\right.
$$

respectively.

$\left(C_{5}\right): f\left(t, u_{1}, \cdots, u_{n-2}, u_{n-1}\right), v(t)$ and $w(t)$ satisfy

$v^{(n-2)}(t) \leq w^{(n-2)}(t)$ on $[0,1]$, and

$f\left(t, v(t), \cdots, v^{(n-3)}(t), u_{n-1}\right)$

$\leq f\left(t, u_{1}, \cdots, u_{n-2}, u_{n-1}\right)$

$\leq f\left(t, w(t), \cdots, w^{(n-3)}(t), u_{n-1}\right)$

for $t \in[0,1],\left(v(t), \cdots, v^{(n-3)}(t)\right) \leq\left(u_{1}, \cdots, u_{n-2}\right) \leq\left(w(t), \cdots, w^{(n-3)}(t)\right)$,

in which

$$
\left(x_{1}, \cdots, x_{n-2}\right) \leq\left(y_{1}, \cdots, y_{n-2}\right) \Longleftrightarrow x_{i} \leq y_{i} \text { for } i=1, \cdots, n-2 .
$$

Remark 2.1. It is clear that

(a) if $f\left(t, u_{1}, \cdots, u_{n-2}, u_{n-1}\right)$ is increasing with respect to $\left(u_{1}, \cdots, u_{n-2}\right)$ on $\mathbb{R}^{n-2}$ for each fixed $\left(t, u_{n-1}\right) \in[0,1] \times \mathbb{R}$, then $\left(C_{5}\right)$ holds;

(b) a simple calculation can show that

$$
\begin{aligned}
& \frac{\partial^{n-2}}{\partial t^{n-2}} K(t, s)=k(t, s) \\
& = \begin{cases}\frac{1}{\rho}(\beta+\alpha s)\{\delta+\gamma(1-t)\}, & 0 \leq s \leq t \leq 1, \\
\frac{1}{\rho}(\beta+\alpha t)\{\delta+\gamma(1-s)\}, & 0 \leq t \leq s \leq 1 ;\end{cases}
\end{aligned}
$$

(c) there exists an $M \in(0,1)$ such that

$$
\left\{\begin{array}{l}
\left(R_{1}\right) \frac{k(t, s)}{k(s, s)} \leq 1, \quad \text { for } t \in[0,1] \text { and } s \in[0,1], \\
\left(R_{2}\right) \frac{k(t, s)}{k(s, s)} \geq M, \quad \text { for } t \in\left[\frac{1}{4}, \frac{3}{4}\right] \text { and } s \in[0,1] .
\end{array}\right.
$$

Now, we can state and prove our main result:

Theorem 2.2 (Main result). Boundary value problem $(B V P)$ has at least one solution $u \in \mathbb{B}$ such that

$$
v^{(i)}(t) \leq u^{(i)}(t) \leq w^{(i)}(t) \text { on }[0,1] \text { for } i=0,1, \cdots, n-2 .
$$


Proof. We separate the proof into the following steps:

Step (1). Consider the modified problem

$\left(\mathrm{BVP}^{*}\right)\left\{\begin{array}{l}\left(E^{*}\right) u^{(n)}(t)+f^{*}\left(t, u(t), u^{(1)}(t), \cdots, u^{(n-2)}(t)\right)=0 \text { for } t \in(0,1), \\ (B C)\left\{\begin{array}{l}u^{(i)}(0)=0,0 \leq i \leq n-3, \\ \alpha u^{(n-2)}(0)-\beta u^{(n-1)}(0)=0, \\ \gamma u^{(n-2)}(1)+\delta u^{(n-1)}(1)=0,\end{array}\right.\end{array}\right.$

where

$$
\begin{aligned}
f^{*}\left(t, u_{1}, \cdots, u_{n-1}\right):=f\left(t, \eta_{1}, \cdots, \eta_{n-1}\right)+\rho\left(\eta_{n-1}-u_{n-1}\right), \\
\eta_{i}:=\left\{\begin{array}{l}
w^{(i-1)}(t) \text { if } u_{i}>w^{(i-1)}(t), \\
u_{i} \text { if } v^{(i-1)}(t) \leq u_{i} \leq w^{(i-1)}(t), \\
v^{(i-1)}(t) \text { if } u_{i}<v^{(i-1)}(t),
\end{array}\right.
\end{aligned}
$$

for all $i=1,2, \cdots, n-1, t \in[0,1]$ and $\rho: \mathbb{R} \longrightarrow[-1,1]$ is the radial retraction defined by

$$
\rho(r):=\left\{\begin{array}{l}
r \text { for }|r| \leq 1 \\
\frac{r}{|r|} \text { for }|r|>1 .
\end{array}\right.
$$

It is clear that (BVP*) has a solution $u=u(t)$ if, and only if, $u$ is the solution of the operator equation

$$
u(t)=\int_{0}^{1} K(t, s) f^{*}\left(s, u(s), u^{(1)}(s), \cdots, u^{(n-2)}(s)\right) d s:=(T u)(t), u \in \mathbb{B},
$$

or

$$
u^{(n-2)}(t)=\int_{0}^{1} k(t, s) f^{*}\left(s, u(s), u^{(1)}(s), \cdots, u^{(n-2)}(s)\right) d s:=(T u)^{(n-2)}(t), u \in \mathbb{B} .
$$

Since $f^{*}$ is continuous and bounded on $[0,1] \times \mathbb{R}^{n-1}, T: \mathbb{B} \longrightarrow \mathbb{B}$ is continuous and compact. Therefore, it follows from Schauder's fixed point theorem (cf. Method 04 ) that $T$ has a fixed point $u \in \mathbb{B}$, i.e. $\left(\right.$ BVP $\left.^{*}\right)$ has a solution $u \in \mathbb{B}$.

Step (2). Let

$$
H(t):=u^{(n-2)}(t)-w^{(n-2)}(t) \text { on }[0,1]
$$

Then we have

$$
\begin{aligned}
& H^{\prime \prime}(\theta) \\
& \geq-f^{*}\left(\theta, u(\theta), \cdots, u^{(n-3)}(\theta), u^{(n-2)}(\theta)\right) \\
& +f\left(\theta, w(\theta), \cdots, w^{(n-3)}(\theta), w^{(n-2)}(\theta)\right) \\
& =-f\left(\theta, \eta_{1}, \cdots, \eta_{n-2}, w^{(n-2)}(\theta)\right)-\rho\left(w^{(n-2)}(\theta)-u^{(n-2)}(\theta)\right) \\
& +f\left(\theta, w(\theta), \cdots, w^{(n-3)}(\theta), w^{(n-2)}(\theta)\right)\left(\eta_{i} \text { is defined as in Step }(1)\right) \\
& \geq-\rho\left(w^{(n-2)}(\theta)-u^{(n-2)}(\theta)\right)>0\left(\text { by using }\left(C_{5}\right)\right)
\end{aligned}
$$

if $\theta \in(0,1)$ such that $H(\theta)>0$.

Therefore, we see that there is no $\theta \in(0,1)$ such that $H(\theta)>0$ and $H^{\prime \prime}(\theta) \leq 0$. 
Step (3). Now, we claim that $H(t) \leq 0$ on $[0,1]$. Suppose to the contrary that there exists a $t_{0} \in[0,1]$ such that $H\left(t_{0}\right)>0$. Then there is a $\theta \in[0,1]$ such that

$$
H(\theta):=\max _{t \in[0,1]} H(t)>0 .
$$

Case(1). Suppose that $\beta=0$, which implies $H(0) \leq 0$ and $\theta \in(0,1]$.

$\left(1^{0}\right)$ Suppose that $\delta=0$, which implies $H(1) \leq 0$ and $\theta \in[0,1)$. Thus, we have $\theta \in(0,1)$ and $H^{\prime \prime}(\theta) \leq 0$. This contradicts the conclusion of Step (2).

$\left(2^{0}\right)$ Suppose that $\gamma=0$, which implies $H^{\prime}(1) \leq 0$. It is clear that $\theta=1$. In fact, if $\theta \in(0,1)$, then $H^{\prime \prime}(\theta) \leq 0$. This gives a contradiction.

Suppose that $H^{\prime}(\theta)=H^{\prime}(1)<0$; then $H(t)$ is strictly decreasing near $t=\theta=1$. This implies $H(\theta)=H(1)$ cannot be the maximum of $H(t)$, thus we obtain $H^{\prime}(1)=$ 0 .

Since $H(\theta)=H(1)>0$, there exists an $\epsilon>0$ such that $H(t)>0$ in $(1-\epsilon, 1]$. Thus $H^{\prime \prime}(t)>0$ in $(1-\epsilon, 1)$, which implies $H^{\prime}(t)$ is strictly increasing on [1- 1,1$]$. It follows from $H^{\prime}(t)<H^{\prime}(1)=H^{\prime}(\theta)=0$ on $[1-\epsilon, 1)$ and we see that $H(1)=H(\theta)$ cannot be the maximum of $H(t)$. This gives a contradiction.

$\left(3^{0}\right) \quad$ Suppose that $\gamma \delta>0$, which implies

$$
w^{(n-1)}(1) \geq \frac{-w^{(n-2)}(1) \gamma}{\delta} \text { and } u^{(n-1)}(1)=\frac{-u^{(n-2)}(1) \gamma}{\delta} .
$$

By Case $(1)-\left(1^{0}\right)$, we see that

$$
u^{(n-2)}(1)>w^{(n-2)}(1) .
$$

Hence, we have

$$
w^{(n-1)}(1) \geq \frac{-w^{(n-2)}(1) \gamma}{\delta}>\frac{-u^{(n-2)}(1) \gamma}{\delta}=u^{(n-1)}(1) .
$$

It follows from Case $(1)-\left(2^{0}\right)$ that we obtain a contradiction.

Case(2). Suppose that $\alpha=0$, which implies $H^{\prime}(0) \geq 0$.

$\left(4^{0}\right)$ Suppose that $\delta=0$, which implies $H(1) \leq 0$ and $\theta \in[0,1)$. It is clear that $\theta=0$. In fact, if $\theta \in(0,1)$, then $H^{\prime \prime}(\theta) \leq 0$. This gives a contradiction.

Suppose that $H^{\prime}(\theta)=H^{\prime}(0)>0$; then $H(t)$ is strictly increasing near $t=\theta=0$. This implies $H(\theta)=H(0)$ cannot be the maximum of $H(t)$, thus we obtain $H^{\prime}(0)=$ 0 .

Since $H(\theta)=H(0)>0$, there exists an $\epsilon>0$ such that $H(t)>0$ in $[0, \epsilon)$. Thus $H^{\prime \prime}(t)>0$ in $(0, \epsilon)$, which implies $H^{\prime}(t)$ is strictly increasing on $[0, \epsilon]$. It follows from $H^{\prime}(t)>H^{\prime}(0)=H^{\prime}(\theta)=0$ on $(0, \epsilon]$ and we see that $H(0)=H(\theta)$ cannot be the maximum of $H(t)$. This gives a contradiction.

$\left(5^{0}\right)$ Suppose that $\gamma=0$, which implies $H^{\prime}(1) \leq 0$. By Case $(1)-\left(2^{0}\right)$ and Case $(2)-\left(4^{0}\right)$, we see that

$$
H(0)>0 \text { and } H(1)>0 .
$$

By continuity and Step (2), we see that there exist $t_{1}, t_{2} \in(0,1)$ such that

$$
t_{1}<t_{2}, H(t)>0, H^{\prime \prime}(t)>0 \text { on }\left(0, t_{1}\right) \cup\left(t_{2}, 1\right) .
$$

It follows from $H^{\prime}(0) \geq 0$ and $H^{\prime}(1) \leq 0$ that

$$
H^{\prime}(t)>0 \text { on }\left(0, t_{1}\right) \text { and } H^{\prime}(t)<0 \text { on }\left(t_{2}, 1\right) .
$$


This implies that there exists a $\theta \in\left(t_{1}, t_{2}\right)$ such that

$$
H(\theta)>0, H^{\prime}(\theta)=0 \text { and } H^{\prime \prime}(\theta) \leq 0,
$$

which contradicts the conclusion of Step (2).

$\left(6^{0}\right) \quad$ Suppose that $\gamma \delta>0$, which implies

$$
w^{(n-2)}(1) \geq \frac{-w^{(n-1)}(1) \delta}{\gamma} \text { and } u^{(n-2)}(1)=\frac{-u^{(n-1)}(1) \delta}{\gamma} .
$$

By Case $(2)-\left(5^{0}\right)$, we see that

$$
u^{(n-1)}(1)>w^{(n-1)}(1) .
$$

Hence, we have

$$
w^{(n-2)}(1) \geq \frac{-w^{(n-1)}(1) \delta}{\gamma}>\frac{-u^{(n-1)}(1) \delta}{\gamma}=u^{(n-2)}(1) .
$$

It follows from Case $(2)-\left(4^{0}\right)$ that we obtain a contradiction.

Case(3). Suppose that $\delta=0$, which implies $H(1) \leq 0$. By Case $(1)-\left(1^{0}\right)$ and Case $(2)-\left(4^{0}\right)$, we see that

$$
\alpha \beta>0 \text { and } u^{(n-2)}(0)>w^{(n-2)}(0) .
$$

Thus, we have

$$
w^{(n-1)}(0) \leq \frac{w^{(n-2)}(0) \alpha}{\beta}<\frac{u^{(n-2)}(0) \alpha}{\beta}=u^{(n-1)}(0) .
$$

It follows from Case $(2)-\left(4^{0}\right)$ that we obtain a contradiction.

Case(4). Suppose that $\gamma=0$, which implies $H^{\prime}(1) \leq 0$. By Case $(1)-\left(2^{0}\right)$ and Case $(2)-\left(5^{0}\right)$, we see that

$$
\alpha \beta>0 \text { and } u^{(n-1)}(0)<w^{(n-1)}(0) .
$$

Thus, we have

$$
w^{(n-2)}(0) \geq \frac{w^{(n-1)}(0) \beta}{\alpha}>\frac{u^{(n-1)}(0) \beta}{\alpha}=u^{(n-2)}(0) .
$$

It follows from Case $(1)-\left(2^{0}\right)$ that we obtain a contradiction.

Case(5). Suppose that $\alpha \beta \gamma \delta>0$. By Case $(1)-\left(3^{0}\right)$, Case $(2)-\left(6^{0}\right)$, Case $(3)$ and Case(4), we see that

$$
\begin{aligned}
& u^{(n-2)}(0)>w^{(n-2)}(0), u^{(n-1)}(0)<w^{(n-1)}(0), \\
& u^{(n-2)}(1)>w^{(n-2)}(1), u^{(n-1)}(1)>w^{(n-1)}(1) .
\end{aligned}
$$

Thus, we have

$$
w^{(n-1)}(0) \leq \frac{w^{(n-2)}(0) \alpha}{\beta}<\frac{u^{(n-2)}(0) \alpha}{\beta}=u^{(n-1)}(0)<w^{(n-1)}(0),
$$

which gives a contradiction.

From Cases(1)-(5), we see that

$$
u^{(n-2)}(t) \leq w^{(n-2)}(t) \text { on }[0,1] .
$$

Similarly, we may show that

$$
v^{(n-2)}(t) \leq u^{(n-2)}(t) \text { on }[0,1] .
$$


Since $u, v, w \in \mathbb{B}$ and satisfy

$$
v^{(n-2)}(t) \leq u^{(n-2)}(t) \leq w^{(n-2)}(t) \text { on }[0,1],
$$

we obtain

$$
v^{(i)}(t) \leq u^{(i)}(t) \leq w^{(i)}(t) \text { on }[0,1] \text { for } i=0,1, \cdots, n-2 .
$$

Therefore,

$$
f^{*}\left(t, u(t), u^{(1)}(t), \cdots, u^{(n-2)}(t)\right)=f\left(t, u(t), u^{(1)}(t), \cdots, u^{(n-2)}(t)\right) \text { on }[0,1] .
$$

That is, $u(t)$ is a solution of (BVP) and satisfies

$$
v^{(i)}(t) \leq u^{(i)}(t) \leq w^{(i)}(t) \text { on }[0,1] \text { for } i=0,1, \cdots, n-2 .
$$

Theorem 2.3 (Main result). Suppose that

(H) there exists a function $g \in\left([0,1] \times[0, \infty)^{n-1} ;[0, \infty)\right)$ which satisfies

$$
\begin{gathered}
f(t, 0,0, \cdots, 0) \geq 0 \text { on }[0,1]\left(f \text { maybe has negative value for } u_{i} \neq 0\right), \\
g\left(t,\left|u_{1}\right|,\left|u_{2}\right|, \cdots,\left|u_{n-1}\right|\right) \geq f\left(t, u_{1}, u_{2}, \cdots, u_{n-1}\right) \text { on }[0,1] \times \mathbb{R}^{n-1}
\end{gathered}
$$

and one of the following:

(2.1) $\max g_{0}=A_{1} \in\left[0, D_{1}\right)$ and $\min g_{\infty}=A_{2} \in\left(\frac{D_{2}}{M}, \infty\right]$,

(2.2) $\min g_{0}=A_{3} \in\left(\frac{D_{2}}{M}, \infty\right]$ and $\max g_{\infty}=A_{4} \in\left[0, D_{1}\right)$,

(2.3) there exist two non-negative functions $h \in C\left([0, \infty)^{n-1} ;[0, \infty)\right)$, increasing with respect to $u_{n-1} \in[0, \infty)$, and $q \in C([0,1] ;[0, \infty))$ such that

$$
\left\{\begin{array}{l}
g\left(t, u_{1}, u_{2}, \cdots, u_{n-1}\right):=q(t) h\left(u_{1}, u_{2}, \cdots, u_{n-1}\right) \quad \text { on }[0,1] \times[0, \infty)^{n-1}, \\
\sup _{u_{n-1} \in(0, \infty)}\left(u_{1}, \cdots, u_{n-2}\right) \in[0, \infty)
\end{array}\right.
$$

where

$$
\begin{gathered}
\max g_{0}:=\lim _{u_{1}, u_{2}, \cdots, u_{n-1} \rightarrow 0^{+}} \max _{t \in[0,1]} \frac{g\left(t, u_{1}, u_{2}, \cdots, u_{n-1}\right)}{u_{n-1}}, \\
\min g_{0}:=\lim _{u_{1}, u_{2}, \cdots, u_{n-1} \rightarrow 0^{+}} \min _{t \in\left[\frac{1}{2}, \frac{3}{4}\right]} \frac{g\left(t, u_{1}, u_{2}, \cdots, u_{n-1}\right)}{u_{n-1}}, \\
\max g_{\infty}:=\lim _{u_{1}, u_{2}, \cdots, u_{n-1} \rightarrow \infty} \max _{t \in[0,1]} \frac{g\left(t, u_{1}, u_{2}, \cdots, u_{n-1}\right)}{u_{n-1}}, \\
\min g_{\infty}:=\lim _{u_{1}, u_{2}, \cdots, u_{n-1} \rightarrow \infty} \min _{t \in\left[\frac{1}{2}, \frac{3}{4}\right]} \frac{g\left(t, u_{1}, u_{2}, \cdots, u_{n-1}\right)}{u_{n-1}}, \\
\left(\int_{0}^{1} k(s, s) d s\right)^{-1}:=D_{1}=\frac{6 \rho}{6 \delta \beta+3 \gamma \beta+\alpha \gamma+3 \alpha \delta}, \\
\left(\int_{\frac{1}{2}}^{\frac{3}{4}} k\left(\frac{1}{2}, s\right) d s\right)^{-1}:=D_{2}=\frac{64 \rho}{16 \beta \delta+6 \beta \gamma+3 \alpha \gamma+8 \alpha \delta}
\end{gathered}
$$

and

$$
Q:=\max _{t \in[0,1]} \int_{0}^{1} k(t, s) q(s) d s .
$$

Then $(B V P)$ has at least one non-negative solution. 
Proof. From the results of Agarwal and Wong [1], [2], [3], we can see that $\left(\mathrm{BVP}^{* *}\right)$ $\left\{\begin{aligned}\left(E^{* *}\right) w^{(n)}(t)+g\left(t, u(t), w^{(1)}(t), \cdots, w^{(n-2)}(t)\right)=0 \text { for } t \in(0,1) \text { and } n \geq 2, & \left\{\begin{array}{l}w^{(i)}(0)=0,0 \leq i \leq n-3, \\ \alpha w^{(n-2)}(0)-\beta w^{(n-1)}(0)=0, \\ \gamma w^{(n-2)}(1)+\delta w^{(n-1)}(1)=0\end{array}\right.\end{aligned}\right.$

has at least one non-negative solution $w(t)$. It is clear that $w(t)$ and $v(t):=0$ are the upper-solution and lower-solution of (BVP), respectively. From Theorem 2.2, we obtain the desired results.

Remark 2.4. For $n=2$, there are many functions $g(t, u)$ that do not satisfy

$$
\max g_{0}, \min g_{0}, \max g_{\infty}, \min g_{\infty} \in\{0, \infty\},
$$

for example, $g(t, u):=\frac{e^{u}-1}{1+t^{2}}\left(\max g_{0}=1\right.$ and $\left.\min g_{0}=\frac{16}{25}\right), g(t, u):=(t+1) \sinh u$ $\left(\max g_{0}=2\right.$ and $\left.\min g_{0}=\frac{3}{2}\right), g(t, u):=u+t^{2} e^{-u}\left(\max g_{0}=\infty, \max g_{\infty}=\right.$ $\left.\min g_{\infty}=1\right)$.

Therefore, our main result generalizes all the recent investigations about the existence of non-negative solutions of (BVP).

\section{ACKNOWLEDGMENT}

The author would like to express his gratitude to the referee for a number of valuable suggestions.

\section{REFERENCES}

[1] R. P. Agarwal and F. H. Wong, Existence of positive solutions for higher order boundary value problems, Nonlinear Studies, to appear.

[2] R. P. Agarwal and F. H. Wong, Existence of positive solutions for non-positive higher order $B V P$ 's, to appear in Computational and Applied Mathematics.

[3] R. P. Agarwal and F. H. Wong, An application of topological transervality with respect to non-positive higher order BVP's, to appear.

[4] V. Anuradha, D. D. Hai and R. Shivaji, Existence results for superlinear semipositive BVP's, Proc. Amer. math. Soc 124 (1996), 757-763. MR 96f:34030

[5] P. B. Bailey, L. F. Shampine and P. E. Waltman, Nonlinear Two-point Boundary Value Problems, Academic Press, New York, 1968. MR 37:6524

[6] C. Bandle and M.K. Kwong, Semilinear elliptic problems in annular domains, Z. Angew. Math. Phys. 40 (1989), 245-257. MR 90m:35062

[7] Y. S. Choi and G. S. Ludford, An unexpected stability result of the near-extinction diffusion flame for non-unity Lewis numbers, Q. J. Mech. Appl. Math 42 part 1 (1989), 143-158. MR 91b:80022

[8] C. J. Chyan and J. Henderson, Positive solutions for singular higher order nonlinear equations, Diff. Eqns. Dyn. Sys 2 (1994), 153-160. MR 97b:34017

[9] E. N. Dancer, On the structure of solutions of an equation in catalysis theory when a parameter is large, J. Diff. Eqns 37 (1980), 404-437. MR 82b:35018

[10] H. Dang and K. Schmitt, Existence of positive solutions for semiliear elliptic equations in annular domain, Diff. and Integ. Equs. 7 (1994), 747-758. MR 94m:35020

[11] K. Deimling, Nonlinear functional analysis, Springer, New York, 1985. MR 86j:47001

[12] J. Dugundji and A. Granas, Fixed point theory, Monografie Mat, PWN, Warsaw, 1982. MR 83j:54038

[13] L. H. Erbe and H. Wang, On the existence of positive solutions of ordinary differential equations, Proc. Amer. Math. Soc. 120 (1994), 743-748. MR 94e:34025

[14] X. Garaizar, Existence of positive radial solutions for semiliear elliptic equations in the annulus, J. Differential Equations 70 (1987), 69-72. MR 89f:35019 
[15] A. Granas, R. B. Guenther and J. W. Lee, On a theorem of S. Bernstein, Pac. Jour. Math. 73 (1977), 1-16. MR 57:10068

[16] A. Granas, R. B. Guenther and J. W. Lee, Nonlinear boundary value problems for some classes of ordinary differential equations, Rocky Mount. J. Math. 10 (1980), 35-58. MR 81h:34017

[17] J. Henderson, Singular boundary value problems for difference equations, Dynamic Systems and Applications 1 (1992), 271-282. MR 94a:39004

[18] P. Kelevedjiev, Existence of solutions for two-point boundary value problems, Nonlinear Analysis T. M. \& A. 22 (1994), 217-224. MR 95a:34029

[19] P. Kelevedjiev, Nonexistence of solutions for two-point boundary value problems, Nonlinear Analysis T. M. \& A. 22 (1994), 225-228. MR 95a:34030

[20] M. A. Krasnoselskii, Positive solutions of operator equations, Noordhoff, Groningen, 1964. MR 31:6107

[21] J. W. Lee and D. O'Regan, Nonlinear boundary value problems in Hilbert spaces, Jour. Math. Anal. Appl. 137 (1989), 59-69. MR 90a:34048

[22] J. Mawhin, Topological degree and boundary value problems for nonlinear differential equations, NSFCB Regional Conference Series in Math., vol. No. 40, Amer. Math. Soc., Providence. R. I., 1979.

[23] N. Vasilev and I. Klokov, Bases of the theory of boundary value problems for ordinary differential equations, Izdat "Zinatne." Riga, 1978.

[24] H. Wang, On the existence of positive solutions for semilinear elliptic equations in the annulus, J. Differential Equations 109 (1994), 1-7. MR 95c:35093

[25] F. H. Wong, Existence of positive solutions of singular boundary value problems, Nonlinear Analysis T.M.\& A. 21 (1993), 397-406. MR 94i:34056

Department of Mathematics and Science, National Taipei Teacher's College, 134, Ho-Ping E. RD. Sec. 2, Taipei 10659, Taiwan, Republic of China

E-mail address: wong@tea.ntptc.edu.tw 